\title{
A comparative study of aqueous DMSO mixtures by computer simulations and integral equation theories
}

\author{
Aurélien Perera ${ }^{1}$ and Bernarda Lovrinčević ${ }^{2}$
}

January 8, 2018

\author{
${ }^{1}$ Laboratoire de Physique Théorique de la Matière Condensée (UMR CNRS \\ 7600), Université Pierre et Marie Curie, 4 Place Jussieu, F75252, Paris cedex 05, \\ France. \\ ${ }^{2}$ Department of Physics, Faculty of Sciences, University of Split, Nikole Tesle \\ 12, 21000, Split, Croatia.
}

\begin{abstract}
Several computer simulation studies of aqueous dimethylsulfoxyde with different force field models, and conducted by different authors, point out to an anomalous depressing of second and third neighbour correlations of the water-water radial distribution functions. This seemingly universal feature can be interpreted as the formation of linear water clusters. We test here the ability of liquid state integral equation theories to reproduce this feature. It is found that the incorporation of the water bridge diagram function is required to reproduce this feature. These theories are generally unable to properly reproduce atom-atom distribution functions. However, the nearideal Kirkwood-Buff integrals are relatively well reproduced. We compute the Xray scattering function and compare with available experimental results, with the particular focus to explain why this data does not reproduce the cluster pre-peak observed in the water-water structure factor.
\end{abstract}




\section{Introduction}

The aqueous dimethyl-sulfoxyde(DMSO) mixtures have been the subject of several studies, mainly because of their peculiar properties at low temperatures [1]. One of the principal interest concerning this mixture is the nature of the waterDMSO interactions, in relation to the low temperature behaviour and cryoprotectancy [2]. Because of this special interest, most of the earlier studies seem to have focused in the water-DMSO clusters, as even very recent studies show the same interest [3, 4, 5, 6, 7, 8]. DMSO has been modeled differently by different authors and there are numerous subsequent computer simulation studies of these particular mixtures[9, 10, 11, 12, 13, 14, 15, 16, 17, 18, 19]. However, despite the various possible water-DMSO force field model combinations, it would appear that all of them indicate that the water-water correlations show the same typical feature, namely a depression of the second and third neighbour correlations. This is illustrated in Fig.1, where the main panel clearly shows that second and third neighbour correlations show oscillations around 1, in contrast with that of aqueous ethanol (dotted line) which is above 1, as usual with aqueous mixtures. Albeit typical variations due to small differences between DMSO models, the universality of this feature is quite visible. While the first peak witness the strong water-water hydrogen bond, which is seen in any mixtures, the depletion of farther neighbours suggests that water has to satisfy 2 constraints: being hydrogen bonded to itself and loose the favoured tetrahedral conformation of bulk water. One solution to this problem is the formation of water string-like clusters. Indeed, such clusters are clearly visible in snapshots from simulations, and at all DMSO mole fractions $x$ in the range $0.1<x<0.9$, as we have been the first to point out their existence [20]. Since various types of experiments show more clearly the existence of water-DMSO bonds [7, 8], one could induce that linear-water clusters must be a consequence of the first more readily visible property. Indeed, all the force field models of DMSO share the fact that the oxygen site is charged negatively while all the other sites, sulfur and the 2 methyl sites, are charged positively. It is quite easy to visualise that the negatively charged water oxygen site would bind to these latter DMSO sites, leaving the 2 hydrogen bonds open to the bulk, hence favouring neighbouring water oxygens to attach to them. This situation will clearly 
disfavour tetrahedral binding of water. This is what computer simulations seem to indicate. This situation is very different from other aqueous mixtures, as for example, water-alcohol mixtures, where water is seen to form globular-like self segregated domains[21]. In Ref.[20], we have argued that it is the large size of the DMSO atom which allows water oxygen to attach to it. However, another puzzle arises: why available Xray scattering data for this mixture[22] does not show a pre-peak, which should arise from the existence of such water clusters. Indeed, the presence of such clusters induces a clear pre-peak at about $k \approx 0.75 \AA^{-1}$ in the water-water structure factor that we have reported in Ref.[20]. Hence, this prepeak should, in principle, contribute to the total scattered intensity. In the present paper, we explain the absence of scattering pre-peak in narrow connection to the concept of domain order introduced in Ref.[23], which leads to a compensation of the positive pre-peak in like species structure factors by the negative pre-peak of the cross species structure factors. This is always the case for many aqueous mixtures, which show the same universal local domain order. In order to break this "symmetry" and induce a scattering pre-peak, it is necessary that the interface separating the segregated water and solute domains acquires particular properties, which would enhance its contribution to the total scattering. This is the case of micelles, for example, for which water is seen to accumulate in the coronary area. We conjecture here that this is the reason why aqueous mixtures of small monools and 1-2-diols do not show scattering pre-peaks, while these tend to appear in triols, which have ester groups near the surfactant $\mathrm{OH}$ head group, and which attract water in the corona of the micelle.

Concerning liquid state integral equation theories (IET) approaches, these have been intensively tested in the early 90's, but have generally lead to disappointing results for associated liquids [24, 25]. To make this point clear, both the molecular IET and the site-site IET are enable to describe the tetrahedral bonding of water through the water-water pair correlation function[25, 26, 27]. The main point we want to make here is that these theories are even less successful for mixtures involving associated liquids, precisely because association induces domain segregation, which is a more complex property to capture. In particular, we want to show that correcting the deficiencies of pure liquid description, by introducing bridge diagram for water, cannot account for the dramatic change in structure of 
segregated mixtures. The particular interest to test these theories for the case of aqueous DMSO, is the fact that this mixtures shows quasi-ideal Kirkwood-Buff integrals, unlike most other aqueous mixtures[28]. In that, this particular system seems to have less concentration fluctuation, and therefore should be more amenable to mean-field like approaches such as IET. In Ref.[20], we have argued that this feature of the aqueous DMSO mixtures was related to water linear aggregates forming pseudo molecular entities, leading to a weakly interacting pseudo ideal mixture of DMSO with these aggregates. Since IET are quiet good for weakly interacting and fully disordered mixtures, it would be interesting to see how much of this property can be captured by these theories. Indeed, there are 2 problems here: the first is low concentration fluctuations, which is favourable for a IET description, and the second is the description of hydrogen bonded aggregates, which should be a challenge for IET.

The remainder of this paper is as follows. In the next section, we briefly remind the models used, simulation protocols and theoretical details related to the IET and their use in the present case. Details concerning the concept of domain order and calculation of the scattering functions are equally gathered in the last subsection. Section 3 details our results for the structural functions and the KIB. Finally, Section 4 gathers our conclusions and perspective on the present work.

\section{Theoretical details}

\subsection{Models and computer simulations}

We have essentially followed the methodology reported in Ref.[20]. Most of the data shown here is from the same simulations presented in this work. Additional structure factors have been computed in order to allow for the calculation of the scattering functions. To summarize, we have used the SPC/E water model[29] and the VB DMSO model[30]. We have also used in Fig.1 the OPLS DMSO model[31]. $N=2048$ particle simulations were used in the isothermal and isobaric molecular dynamics simulations with the Gromacs package[32]. Specific details can be found in Ref.[[20]]]. 


\subsection{Integral equation theory}

We have used the site-site IET formalism, which has the same level of accuracy as the molecular version, as far as associated liquids are concerned. Although one would expect a superior accuracy of the molecular formulation, due to an exact version of the molecular Ornstein-Zernike (MOZ) equation[33, 34], instead of the approximate site-site Ornstein-Zernike (SSOZ) version[24, 34], it is the constraint imposed by closure relation which dominates the structural description. Indeed, the description of hydrogen bonded structures in the associated liquids is particularly constraining, requiring 3-body and higher order correlations present in the missing highly connected and irreducible bridge diagrams[34]. This is the reason for the similarity in the poor description of both approaches for water from MOZ[26] and SSOZ[27].

The 2 equations to solve are the site-site $\mathrm{OZ}$ matrix equation together with a closure equation. The SSOZ equation reads

$$
S . M=I
$$

where

$$
S=W+\rho H
$$

is the generalized structure factor matrix, with elements defined as

$$
S_{i_{\alpha j_{\beta}}}(k)=W_{i_{\alpha} j_{\beta}}(k)+\rho H_{i_{\alpha} j_{\beta}}(k)
$$

where the index $i_{\alpha}$ and $j_{\beta}$ designate atom $\mathrm{i}$ of molecular species $\alpha$ and atom $\mathrm{j}$ of molecular species $\beta$, respectively, and

$$
M=W^{-1}-\rho C
$$

The matrix $W$ describes the intramolecular correlation, defined as rigid atomic bonds within molecular species

$$
W_{i_{\alpha} j_{\beta}}(k)=\frac{\sin \left(k d_{i_{\alpha} j_{\alpha}}\right)}{k d_{i_{\alpha} j_{\alpha}}}
$$


where $d_{i_{\alpha} j_{\alpha}}$ is the distance between atomic sites $i_{\alpha}$ and $j_{\alpha}$ of molecule of species $\alpha$. The matrix $\mathrm{H}$ is related to the site-site distribution functions $g_{i_{\alpha} j_{\beta}}(r)$, with $h_{i_{\alpha} j_{\beta}}(r)=g_{i_{\alpha} j_{\beta}}(r),-1$, and $\tilde{h}_{i_{\alpha} j_{\beta}}(k)=\int d \vec{r} h_{i_{\alpha} j_{\beta}}(r) \exp (\overrightarrow{i k} . \vec{r})$ the Fourier transform of $h_{i_{\alpha} j_{\beta}}(r)$, and the matrix $\mathrm{C}$ is related to the Fourier transform of the site-site direct correlation function $c_{i_{\alpha} j_{\beta}}(r)$ with the relations

$$
\begin{aligned}
H_{i_{\alpha} j_{\beta}} & =\sqrt{x_{\alpha} x_{\beta}} \tilde{h}_{i_{\alpha} j_{\beta}}(k) \\
C_{i_{\alpha} j_{\beta}} & =\sqrt{x_{\alpha} x_{\beta}} \tilde{c}_{i_{\alpha} j_{\beta}}(k)
\end{aligned}
$$

where $x_{\alpha}$ is the mole fraction of species $\alpha$.

For the closure equations, we have chosen both the hypernetted chain (HNC) equation and the Kovalenko-Hirata $(\mathrm{KH})$ closure [35]. The HNC closure equation reads

$$
g_{i_{\alpha} j_{\beta}}(r)=\exp \left(-\beta v_{i_{\alpha} j_{\beta}}(r)+h_{i_{\alpha} j_{\beta}}(r)-c_{i_{\alpha} j_{\beta}}(r)+b_{i_{\alpha} j_{\beta}}(r)\right)
$$

where $v_{i_{\alpha} j_{\beta}}(r)$ is the interaction between the atomic sites $i_{\alpha}$ and $j_{\beta}\left(\beta=1 / k_{B} T\right.$ is the Boltzmann factor), and the last term $b_{i_{\alpha} j_{\beta}}(r)$ represents the so-called bridge function. Setting $b_{i_{\alpha} j_{\beta}}(r)=0$ leads to the strict HNC closure, while this function can also be tailored from the site-site correlation functions obtained through the procedure we have outlined in Ref.[36].

The KH equation is a mix between the strict $\mathrm{HNC}$ equation above and the mean spherical approximation, based on the value of the term $\Gamma_{i_{\alpha} j_{\beta}}(r)=-\beta v_{i_{\alpha} j_{\beta}}(r)+$ $h_{i_{\alpha} j_{\beta}}(r)-c_{i_{\alpha} j_{\beta}}(r)$ in the exponential of Eq. 6 ;

$$
g_{i_{\alpha} j_{\beta}}(r)= \begin{cases}\exp \left(\Gamma_{i_{\alpha} j_{\beta}}(r)\right) & \text { when } \Gamma_{i_{\alpha} j_{\beta}}(r)<0 \\ 1+\Gamma_{i_{\alpha} j_{\beta}}(r) & \text { when } \Gamma_{i_{\alpha} j_{\beta}}(r)>0\end{cases}
$$

This ad-hoc procedure of damping the raise of the correlations in the exponential through the term $\Gamma_{i_{\alpha} j_{\beta}}(r)$ allows to control its dramatic growth in the case of $\mathrm{HNC}$, which leads to the no-solution and spinodal-like scenario often spuriously encountered in the numerical solution of these approximate closures. We will discuss the occurrence of this type of scenario in the present case, below in the results 
sub-sections.

Finally, we have equally examined the Percus-Yevick (PY) closure, which is a linearised version of $\mathrm{HNC}$

$$
g_{i_{\alpha} j_{\beta}}(r)=\exp \left(-\beta v_{i_{\alpha} j_{\beta}}^{(S R)}(r)\right)\left[1++h_{i_{\alpha} j_{\beta}}(r)-c_{i_{\alpha} j_{\beta}}^{(S R)}(r)\right]
$$

where the superscript (SR) indicates that only the short range interactions and direct correlations are considered. The long range Coulomb part is removed from both functions following a standard procedure described in the literature[37]. In fact, this is also the case in the $\mathrm{HNC}$ and $\mathrm{KH}$ closures, because of the exact limiting relation:

$$
\lim _{r \rightarrow \infty} c_{i_{\alpha} j_{\beta}}(r)=-\beta v_{i_{\alpha} j_{\beta}}(r)
$$

which allows to retain only the short range part of the direct correlation function in the various closure equations.

As shown in the Results section, both the HNC and the PY equations cannot be solved for the mixture case, precisely because of the behaviour of the term $h_{i_{\alpha} j_{\beta}}(r)-c_{i_{\alpha} j_{\beta}}^{(S R)}(r)$, which induces the growth of medium range correlations, which, in turn, promotes spurious phase separation-like behaviour. The damping procedure of the $\mathrm{KH}$ closure appears then as a wise method. However, we will discuss below the appropriateness of this artificial method in view of the physical phenomena in hydrogen bonding mixtures.

Both the SSOZ equation and the closure equations are solved iteratively for the unknown site-site functions $h_{i_{\alpha} j_{\beta}}(r)$ and $c_{i_{\alpha} j_{\beta}}(r)$. These functions are discretised on a grid of 2048 points, with r-spacing of $\Delta r=0.03 \AA$.

We find that the KH closure yields numerical solution for the aqueous DMSO mixtures for all concentrations at room temperature. Conversely, neither the PY nor the HNC closures are able to yield numerical solutions at $T=300 \mathrm{~K}$. While we do not expect that the linearised PY closure to be able to handle complex structures, the HNC closure deserves some comments.

The strict HNC closure is unable to yeald numerical solution below very high temperatures around $T=5000 \mathrm{~K}$ for the equimolar mixture. At such elevated temperature, HNC predicts high small-k raise of the structure factors, although no obvious long range tail seem to develop in the site-site correlations. This 
is a pathology of HNC closure, as noted early in the historical development of IET[38]. The mathematical origin of such behaviour is unclear at present. It is generally admitted that this behaviour underlies a spurious spinodal[39]. However, as we have mentioned above, we do not observe that any corresponding long range tail develops. At such high temperature where we can find numerical solutions, the site-site functions are nearly structureless, just like expected for any thermally disordered fluid. In fact, comparing with the KH closure results at the same temperature reveals that the first neighbour water-water correlations are overestimated in the HNC closure, leading to the $k=0$ increase. In other words, it is the short range behaviour of the correlation which leads to the $k=0$, and not a spinodal behaviour, contrary to the generally accepted idea about this closure.

\subsection{Domain order and the scattering function}

The X-ray scattering intensity $I(k)$ can be obtained through the Debye formula[40]

$$
I(k)=<\sum_{i_{\alpha}, j_{\beta}} f_{i_{\alpha}}(k) f_{j_{\beta}}(k) \exp \left(i \mathbf{k} .\left(\mathbf{r}_{i_{\alpha}}-\mathbf{r}_{j_{\beta}}\right)\right)>
$$

where the sum runs over all pairs of scattering atoms $i_{\alpha}$ and $j_{\alpha}$, which are at respective spatial positions $\mathbf{r}_{i_{\alpha}}$ and $\mathbf{r}_{j_{\beta}}$, the functions $f_{i_{\alpha}}(k)$ are the atomic form factor for atom $i_{\alpha}$ and depend on the type of radiation which is scattered (we use here Xray), and the symbol <...> designates an average over all possible positions of these atoms, which corresponds to a thermal average, or an ensemble average for calculational purposes. It can be shown[23, 41] that this expression can be rewritten in a form which can be used in theoretical methods

$$
I(k)=I_{\text {Ideal }}(k)+I_{\operatorname{Intra}}(k)+I_{\text {Inter }}(k)
$$

where the 3 contribution represent, respectively the ideal part

$$
I_{\text {Ideal }}(k)=\sum_{\alpha} x_{\alpha} \sum_{i_{\alpha}} f_{i_{\alpha}}(k)
$$

the intra-molecular part 


$$
I_{\text {Intra }}(k)=\sum_{\alpha \beta} \sqrt{x_{\alpha} x_{\beta}} \sum_{i_{\alpha} j_{\beta}} f_{i_{\alpha}}(k) f_{j_{\beta}}(k) W_{i_{\alpha} j_{\beta}}(k)\left(1-\delta_{i_{\alpha j} j_{\alpha}} \delta_{\alpha \beta}\right)
$$

where $W_{i_{\alpha} j_{\beta}}(k)$ is given by Eq. $(5)$, and inter-molecular part

$$
I_{\text {Inter }}(k)=\sum_{\alpha \beta} \sqrt{x_{\alpha} x_{\beta}} \sum_{i_{\alpha} j_{\beta}} f_{i_{\alpha}}(k) f_{j_{\beta}}(k) H_{i_{\alpha} j_{\beta}}(k)
$$

The Kronecker delta terms in Eq. (12) avoid the self-atomic contributions inside same species; in which case it is the ideal term in Eq. (11) which prevails. The ideal contribution corresponds to non-interacting and non-bonded atoms. The "intra" part brings in the fact that atoms are bonded into molecules. Finally the "inter " part accounts for the correlations, hence interaction between atoms and molecules.

The Xray intensity, as written through Eqs.(10-13), can be calculated for both the cases of simulations and IET, since the correlation functions $g_{i_{\alpha} j_{\beta}}(r)$ are available.

\section{Results}

\subsection{Chain-like water clusters}

As shown in Ref.[20], the computer simulations of different combinations of model water and model DMSO indicate that water tends to form predominantly chain-like clusters. This is illustrated in Fig.1. This feature is not the one which is usually reported in the literature, where the accent is more on the water-DMSO dimer formation. However, these two properties are not necessarily excluding each other. What is interesting is to know which property induces the other. One view point is that, water tends to bind preferentially to water, which then dictates subsequently all other structural features. This property is reflected in the model simulation through the fact that the partial charges on the oxygen of water are quite high ( $q_{O_{W}} \approx-0.85$ for the oxygen atom, for almost all water models), and higher than for most solutes (for DMSO, the charge on the oxygen atom is $q_{O_{D}} \approx-0.46$ and on the sulfur atom $q_{S_{D}} \approx 0.14$, for acetone $q_{O_{A}} \approx-0.56$, and for mono-ols 
$\left.q_{O} \approx-0.7\right)$. A logical consequence of electrostatic is that charges with highest valence will tend to dominate the interactions, leading weaker charges to adapt. Following this line of reasoning, water is more likely to bind with itself, and preserve pockets of tetrahedrally bound water molecules. This is what happens with monools [42] and acetone [43]. We would then expect something similar with DMSO as well, and at even greater scale, since the valence is smaller. This is not what sis observed in Fig.1: water seems to mix rather well with DMSO, precisely by forming chain-like clusters. Clearly, the competition between charges alters the simplistic picture presented above. High valences on the solute equally favours self solute binding, thereby enforcing the micro-segregation observed in simulation of aqueous-acetone [43] or aqueous-alcohol[23, 42]. Intuitively, weaker charges on the solute should increase water segregation. This is what we observed in aqueous mixtures of "weak-water" models, where the valence of the latter weak-water was decreased to various values. Therefore, the reason for the enhanced miscibility of water in these mixtures is to be searched elsewhere.

\subsection{Structure functions}

We look at the site-site correlation functions and associated structure factors of pure DMSO and aqueous DMSO, as obtained from computer simulations and IET.

\subsubsection{Pure water}

The case of pure water has been reported in several previous works by many authors[24, 26, 27], including our own work [36]. The comparison with scattering experiments shows that classical force field such as SPC/E are quite adequate. However, structure prediction from IET cannot capture the strong tetrahedral ordering of water, and predict a structure closer to that of a simple Lennard-Jones liquid. Interestingly, and as stated above, both the molecular and the site-site produce very similar correlations, which indicates that the principal issue is not in the differences between the two methods, but the fact they both miss many body correlations. In the present work, the water-water correlations for mixtures are corrected by the pure water bridge terms, which we have computed in Ref.[36]. 
We show that this contribution is capital to produce the chain-like water clusters within the HNC theory.

\subsubsection{Neat DMSO}

Fig.2 shows selected atom-atom correlation functions and corresponding structure factors for pure DMSO, as obtained from simulations (curve in green), HNC (blue), $\mathrm{KH}$ (red dashes) and PY(magenta). It is seen that the first neighbor correlations are generally underestimated by the theories, but not dramatically so. The PY theory is not able to properly describe the features of the structure factors. But the principal problem with the theories is seen in small-k behaviour of the structure factors, which all show a pronounced raise, suggesting liquid-vapour pre-transitional prediction. This is most serious for the HNC closure. A close look at the long range behaviour of the correlation functions shows a general tendency to be above the asymptote 1 .

\subsubsection{Aqueous-mixtures}

Selected correlations between the oxygen atoms of water and DMSO molecules, as well as associated structure factors, are displayed for typical DMSO mole fractions $x=0.1,0.5$ and 0.8 , in Fig.3, Fig.4 and Fig.5, respectively. The color conventions are similar to Fig.2, simulation results in green, $\mathrm{HNC}$ in blue and $\mathrm{KH}$ in red dashes. What is immediately apparent is how poor IET results are. Despite the fact that water-water correlations contain the bridge function which reproduces pure water data accurately, it is not sufficient to enforce a better agreement than the $\mathrm{KH}$ closure. In fact, this closure seems to reproduce the trends of the correlations for the $10 \%$ DMSO case in Fig.4a, while the enhanced HNC is very poor. The situation is somewhat improved for the 50\% and 80\% DMSO cases. Perhaps the most important feature comes from the structure factors in the small-k region, where the enhanced $\mathrm{HNC}$ version tends to predict the cluster pre-peak visible on the simulation data at all concentrations. The $\mathrm{KH}$ approximation, despite being somewhat more accurate, predicts a small-k raise at $\mathrm{k}=0$ for the water-water correlations. In fact, the strict HNC closure cannot be solved for this mixture at room temperature, precisely because of spurious $\mathrm{k}=0$ raise in the correlations. The lin- 
earisation present in the $\mathrm{KH}$ closure prevents this raise to be happen in the same dramatic fashion that for the strict HNC closure, but it is at the heart of these approximations limited to pair correlations. It is only through the introduction of higher order correlation that this problem can be cured. This fact indicates how crucial such correlations are to represent the complexity of associating mixtures.

As indicated in our previous study of aqueous-DMSO mixtures, the most important feature of the correlations is the presence of a pre-peak in the water-water structure factors, in the k-vector range $k \approx 0.7-1 \AA^{-1}$. This is due to chain-like water aggregates, as indicated by the second and third neighbour depression in the water-water correlations. These positive pre-peak contributions are accompanied with negative pre-peak contributions in the water-DMSO cross correlations. This was clearly illustrated in Fig.7 of Ref.[20]. The presence of positive and negative pre-peaks is not specific to the present system, and are equally found in room temperature ionic liquids[44]. They correspond in fact to an underlying charge ordering between positively and negatively charged atomic groups. Despite the strong local inhomogeneity induced by such charge order, which is responsible for the micro-heterogeneity as well as the linear water aggregates found here, the global homogeneity is preserved. A signature of this global homogeneity is the perfect compensation of these positive and negative pre-peak in the scattered intensity, as we show in sub-section 4 below.

\subsection{Charge order}

An important property of the structure of neat DMSO is revealed in Fig.6. It shows that the long range correlations between the oxygen and sulfur atoms with methyl atoms are out-of-phase with all the types of atom-atom the correlations. This property is typical of charge ordering[45], as recently advertised in room temperature ionic-liquids [46, 47]. Charge order essentially describes the alternated disposition of positive and negatively charged atoms, most appropriately in ionic melts. But this order can equally occur between charged atomic groups in complex room temperature ionic liquids [46, 47]. The hydrogen bonding in associative liquids is such a nice example of charge ordering, alternating oxygen and hydrogen atoms, which in this case enforce the tetrahedral ordering in water, or 
the chain-like ordering in mono-ols. Therefore, what we observe in DMSO is an alternance of oxygen and methyl. We conjecture here that it is this ordering which imposes the ordering of the water molecules, which then are not obliged to cluster into globular clusters, but rather meander between the DMSO molecules through linearly bound water chains. Fig. 3 equally shows that none of the theories are able to describe the strength of this charge ordering within the same spatial range, and considerably underestimate it. This could be due to high order diagrams missing. We did not try incoporating such diagrams by the procedure which we used in other cases, for the reason explained in the next sub-section.

\subsection{Kirkwood-Buff integrals}

Fig.7 shows the KBI from experiments (filled dots) compared with simulations (open triangles) and IEt results, HNC (full lines) and $\mathrm{KH}$ ( dashed lines). We note that generally, the agreement between theoretical and experimental results are rather good. This is even more surprising when the IET results for structure are quite disappointing. We note that the $\mathrm{KH}$ results are stunning accurate compared to the experiments, except for $G_{D D}$ for small DMSO content. The enhanced HNC results are worse for $G_{W W}$ at high DMSO content. In fact, they seem to follow the results of the simulations rather well, which is intriguing. Indeed, the force field model we have used are exactly the same for both theoretical methods. Despite these shortcomings, this thermodynamic property related to structure is rather well reproduced. This is in sharp contrast with the KBI results for other aqueous mixtures, such as water- alcohol, for example, for which the KBI from IET are very poor. Comparing the nature of the aggregation in both systems, since aqueous alcohol mixtures tend to present quite large water-water KBI in relation with strong micro-heterogeneity and globular water aggregates, while aqueous DMSO has rather small micro-heterogeneity and linear water aggregates, it would seem that IET are better suited for weakly aggregating mixtures. This is inline with our previous work with aqueous weak-water mixture models[48]. 


\subsection{Scattering function}

Fig.8a-b show the Xray scattering intensity from all 3 theoretical methods compared with experimental data for pure water and pure DMSO. For water, the full intensity is represented. For DMSO, another common representation is often used by experimentalists and simulators is:

$$
\Delta I(k)=k \cdot\left(I(k)-I_{\text {ideal }}(k)\right)
$$

It can be seen that, despite minor discrepancies in the correlation functions, the IET manage to reproduce the total scattered intensity rather well.

Fig.9a-c show the scattering intensity for mixtures, compares with the Xray data from Nishikawa[22]. We note that the simulations are in good agreement with the experimental data, and that the IET perform quite well, specially when compared with the rather poor structure factors shown in Figs.4. This is certainly due to favourable compensations in the sum of Eq.(13).

Perhaps the most important feature is the disappearance of the water pre-peak from the total intensity. This is due to a perfect compensation from the waterwater and water-DMSO cross contributions in Eq.(13). The positive pre-peak of the first are exactly compensated by the negative contributions from the second. This can be seen in the partial contributions shown in the insets.

The insets of the figures show the total scattered intensity $I(k)$, together with other contributions in Eq. 10 , namely $I_{\text {ideal }}(k)$ in green line and $\Delta I_{\text {Intra }}(k)=$ $I_{\text {Intra }}(k)-I_{\text {Ideal }}(k)$ in jade. The water-water and water-DMSO cross contributions are equally shown in cyan and magenta, respectively. These 2 curves serve to illustrate how the water aggregate pre-peak gets compensated into the total intensity $\mathrm{I}(\mathrm{k})$ (in blue). It is equally important to realize that the intra-molecular contribution is quite important, and needs to be added to the intermolecular contribution which is solely made of the site-site functions.

\section{Discussion and Conclusion}

The present work illustrates how approximate IET, which are obviously unable to account for the local ordering due to association in aqueous mixtures, can however 
turn out to be relatively acceptable when computing averaged quantities accessible to experiments, such as the KBI or the radiation scattering intensity. The first concerns a spatial average, and is related to various thermodynamic properties [49] , while the second is an average through summing over all site-site contributions. IET are relatively accurate in many situations, such as simple liquids and their mixtures[?], weakly polar systems[?], ionic melts[56]. The present results indicate that their accuracy comes essentially when 2-body interactions and correlations are predominant. If clustering is present, then these theories become useless. A supplementary hint comes from the fact that IET are very unreliable to describe critical fluctuations, which means in the vicinity of phase transitions[39]. The HNC theory is not even able to predict a spinodal behaviour[39]. Since aggregation is a k-dependent concentration fluctuation, the latter which simply the $\mathrm{k}=0$ part, we do not expect these theories to be able to describe directional clustering properly. This is the principal reason of the problems reported here. Nevertheless, the fact that they can be used for weakly aggregating systems, such as aqueousDMSO, indicate the importance of many body correlations in various other aqueous mixtures. The multiple recipe to improve IET for spherical interaction are of no use when directional clustering is present. The present work, equally indicates that accounting for pure water association is not enough to permit to describe what happens in the case of mixtures. The no-spinodal pathology of the HNC closure indicates that recepy such as the KH closure do not bring any deep insight into the relation between directional molecular association and many body correlations. Insights into this matter are still missing.

It is equally interesting to see that radiation scattering, ore specifically Xray scattering, does not account for the local structure of the aqueous-DMSO mixtures, namely the linear chain aggregates. While this is quite apparent from the water-water correlations in many model combinations, it is not immediately deducible from spectroscopy data, which tends to enhance water-DMSO pairing, and even less from scattering data, where the pre-peak is totally absent. In a way, this result indicates that computer simulations provide a better insight into the local microscopic structure than experimental radiation scattering can provide. Perhaps neutron scattering experiments can provide additional insight into this matter, through various deuteration processes. This point remains open for 
subsequent investigations.

\section{References}

[1] B. Kirchner and M. Reiher, J. Am. Chem. Soc. 124, 6206 (2002)

[2] J. B. Mandumpal, C. A. Krek and R. L. Mancera, Phys. Chem. Chem. Phys. 13, 3839 (2011)

[3] I. A. Borin and M. S. Skaf, J, CHem. Phys. 110, 6412 (1999)

[4] B. Kirchner, D. J. Searles, A. J. Dyson, P. S. Vogt and H. Huber, J. Am. Chem. Soc. 122 , 5379 (2000)

[5] N. Engel, K. Atak, K. Lange, M. Gotz, M. Soldatov, R. Golnak, E. Suljoti, J.-E. Rubensson and E. F. Aziz, J. Phys. Chem Lett. 3, 3697 (2012)

[6] P. P. Wiewior, H. Shirota, and E. W. Castner, Jr, J. CHem. Phys. 116, 4643 (2002)

[7] N. Engel et al., J. Phys. Chem. Lett. 3, 3697 (2012)

[8] K. Oh, K. Rajesh, J. F. Stanton and C. R. Baiz, Angew. Chem. Int. Ed. 56, 11375 (2017)

[9] B. G. Rao and U. C. Singh, J. Am. Chem. Soc. 112, 3803 (1990)

[10] I. I. Vaisman and M. L. Berkowitz, J. Am. Chem. Soc. 114, 7889 (1992)

[11] A. Luzar and D. Chandler, J. Chem. Phys. 98 , 8160 (1993)

[12] A. Luzar, A. K. Soper and D. Chandler, J. Chem. Phys. 99 , 6836 (1993)

[13] H. Liu, F. Müller-Plathe and W. F. van Gunsteren, J. Am. Chem. Soc. 117, 4363 (1995) 
[14] E. Hawlicka, P. J. Chem. 70, 821 (1996)

[15] A. Vishyakov, A. P. Lyubartsev and A. Laaksonen, J. Phys. Chem. A105, 1702 (2001)

[16] Y. Lei, H. Li and S. Han, Chem. Phs. Lett. 380 , 542 (2003)

[17] D. P. geerke, C. Oostenbrink, N. F. A. van der Vegt and W. F. van Gunsteren, J. Phys. Chem. B108, 1436 (2004)

[18] S. E. McLain, A. K. Soper and A. Luzar, J. Chem. Phys. 127, $174515(2007)$

[19] M. R. Harpham, N. E. Levinger and B. M. Ladanyi, J. Phys. Chem. B112, 283 (2008)

[20] A. Perera and R. Mazighi J. Chem. Phys. 143, 154502 (2015)

[21] A. Perera, Pure and Appl. Chem. 88 ,189 (2016)

[22] Y. Koga, Y. Kasahara, K. Yoshino and K. Nisihikawa, J. Sol. Chem. 30, 885 (2001)

[23] A. Perera, Phys. Chem. Chem. Phys. 19, 1062 (2017)

[24] L. Lue and D. Blanckstein, J. Phys. Chem. 96, 8582 (1992)

[25] L. Lue and D. Blanckstein, J. Chem. Phys. 102, 5427 (1994)

[26] M. Lombardero, C. Martin, S. Jorge; F. Lado and E. Lomba, J. Phys. Chem. 110, 1148 (1999)

[27] B. M. Pettit and P. J. Possky, J. Chem. Phys. 77, 1451 (1982)

[28] E. Matteoli and L. Lepori, J. Chem. Phys. 80, 2856 (1984)

[29] J. C. Berendsen, J. P. M. Postma, W. F. Von Gusteren and J. Hermans, in Intermolecular Forces, edited by B. Pullman (Reidel, Dortrecht, 1981) 
[30] I. I. Vaisman and M. L. Berkowitz, J. Am. Chem. Soc. 114, 7889 (1992)

[31] W. L. J. Jorgensen. Phys. Chem. 90, 6379 (1986)

[32] D. van der Spoel., E. Lindahl, B. Hess., G. Groenhof, A. E. Mark and H. J. C. Berendsen, 2005, J.Comp. Chem. 26, 1701

[33] P. G. Kusalik and G. N. Patey, Mol. Phys. 65, 1105 (1988)

[34] J. P. Hansen and I. R. McDonald, Theory of Simple Liquids (Academic, London, 1986)

[35] A. Kovalenko and F. Hirata, Chem. Phys. Lett. 349, 496 (2001).

[36] B. Kežić and A. Perera, J. Chem. Phys. 135, 234104 (2011)

[37] K.-C. Ng, J. Chem. Phys. 61, 2680 (1974)

[38] M. I. Guerrero, G. Saville and J. S. Rowlinson, Mol. Phys. 29, 1941 (1975)

[39] C. Caccamo, Phys. Rep. 274, 1 (1996)

[40] P. Debye, Ann. Phys. 351, 80923 (1915).

[41] C. J. Pings and J. Waser, J. Chem. Phys., 48, 3016 (1968).

[42] B. Kežić and A. Perera, J. Chem. Phys. 137, 014501 (2012)

[43] B. Kežić and A. Perera, J. Chem. Phys. 137, 134502 (2012)

[44] A. Perera and R. Mazighi , J. Mol. Liq. 210, 243 (2015)

[45] A. Perera , Phys. Chem. Chem. Phys. 19, 1062 (2017)

[46] C. S. Santos, H. V. R. Annapuredy, N. S. Murthy, H. K. Kashyap, E. W. Castner Jr. and C. J. Margulis, T, J. Chem. Phys. 134, 064501 (2011) 
[47] Y. Wang, W. Jiang, T. Yan and G. A. Voth , Acc. Chem. Res. 40, 1193 (2007)

[48] A. Perera, R. Mazighi, B. Kežić and P. N. Phuong, Mol. Phys. 112, $1262(2014)$

[49] J. G. Kirkwood and F. Buff, J. Chem. Phys. 19, 774 (1950)

[50] F. J. Rogers and D. A. Young, Phys. Rev. A 30, 999 (1984)

[51] P. T. Cummings and P. A. Monson, J. Chem. Phys. 82, 4303 (1985)

[52] L. L. Lee and A. Malijevsky, J. Chem. Phys. 114, 7109 (2001)

[53] J. M. Eggebrecht, D. J. Isbister and J. C. Rasaiah, J. Chem. Phys. 73, 3980 (1980)

[54] F. Hirata and P. J. Rossky, Chem. Phys. Lett. 83, 329 (1981)

[55] P. H. Fries and G. N. Patey, J. Chem. Phys. 82, 429 (1985)

[56] M. Baus and J. P. Hansen, Phys. Rep. 59, 1 (1980)

[57] J. M. Sorenson, G. Hura, R. M. Glaeser and T. Head-Gordon, J. Chem. Phys. 113, 9149 (2000)

[58] M. Mijaković , K. D. Polok, B. Kežić , F. Sokolić , A. Perera and L. Zoranić Molecular Simulation 42 , 699 (2014) 


\section{Figure captions}

Fig.1 Comparison between various water-water RDF reported in the literature for aqueous-DMSO mixtures. Blue line is pure SPC/E water, red line is $\mathrm{x}=0.35$ from Ref.[11], green line for $\mathrm{x}=0.33$ from Ref.[15], jade line is for $\mathrm{x}=0.3$ from Ref.[20], orange line from OPLS DMSO, and dotted gray line for aqueous ethanol $x=0.3[58]$.

Fig. Snapshot of the 50\% aqueous DMSO mixture (2048 particles) evidencing linear water clusters. Water molecules are shown as red ball for oxygen atom and white for hydrogen atoms. DMSO is shown in cyan semi-transparent.

Fig.3 Selected atom-atom correlation functions (a) and corresponding structure factors (b) for the neat DMSO mixture, comparing simulation data (green) to $\mathrm{HNC}($ blue), $\mathrm{KH}$ (dashed red) and PY(thin magenta) data. The various atom pairs are indicated in the panels.

Fig.4 Oxygen atom correlation functions (a) and associated structure factors (b) for the $10 \%$ aqueous DMSO mixture. The color conventions are as in Fig.2.

Fig.5 Oxygen atom correlation functions (a) and associated structure factors (b) for the $50 \%$ aqueous DMSO mixture. The color conventions are as in Fig.2.

Fig.6 Oxygen atom correlation functions (a) and associated structure factors (b) for the $80 \%$ aqueous DMSO mixture. The color conventions are as in Fig.2.

Fig.7 DMSO atom-atom correlation functions demonstrating the long range charge order behaviour (see text). $g_{O O}(r)$ in blue, $g_{S S}(r)$ in purple, $g_{S M}(r)$ in black, $g_{M M}(r)$ in green, $g_{O S}(r)$ in red and $g_{O M}(r)$ in orange. The main panel is for neat DMSO, the upper two insets for $80 \%$ and $50 \%$ aqueous DMSO mixtures, and the lower inset for the HNC results. 
Fig.8 Kirkwood-Buff integrals of the aqueous-DMSO mixtures. $G_{W W}$ in blue, $G_{D D}$ in green and $G_{W D}$ in magenta. Filled dots are experimental results from Ref.[28], open triangles simulation results from Ref.[20], blue curve for HNC and dashed curves for $\mathrm{KH}$.

Fig.9 Xray scattering intensities $I(k)$ for neat water (a) and neat DMSO (b), together with the $\Delta I(k)$ representations from Eq. 14 Experimental results in red, simulation in blue, IET results in dotted cyan. The ideal contribution from Eq. (11) is in green and the intra-molecular from Eq. (12) contribution in jade. For water, the experimental data for $I(k)$ is from Ref.[57], and $\Delta I(k)$ for both water and DMSO are from from Ref.[22].

Fig.10 Xray scattering intensities $\Delta I(k)$ (main panel) and $I(k)$ (inset) for aqueous mixtures, with $17 \%$ (a), 50\% (b) and $80 \%$ (c) DMSO contents. Experimental results (from Ref.[22]) in red, simulation in blue, $\mathrm{HNC}$ results in cyan, and $\mathrm{KH}$ in dashed jade. The ideal and intramolecular contributions are shown in the insets in dashed green and jade, respectively. The purple curve represents the water-water contribution to $\mathrm{I}(\mathrm{k})$, while the magenta curve is for the cross water-DMSO contribution (see text) 


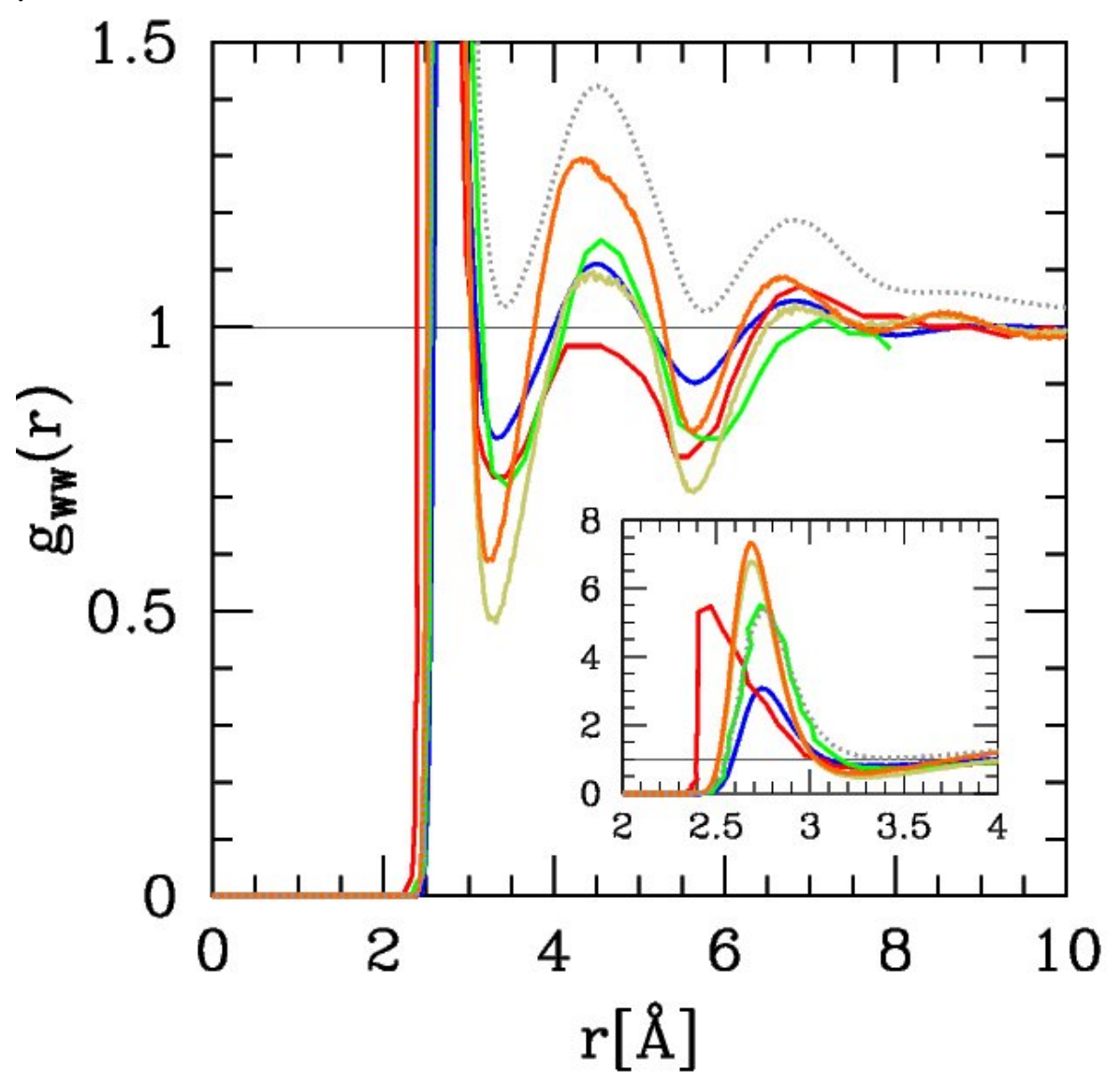

.Fig.1 - Comparison between various water-water RDF reported in the literature for aqueous-DMSO mixtures. Blue line is pure SPC/E water, red line is $\mathrm{x}=0.35$ from Ref.[11], green line for $\mathrm{x}=0.33$ from Ref.[15], jade line is for $\mathrm{x}=0.3$ from Ref.[20], orange line from OPLS model[31], and dotted gray line for aqueous ethanol $\mathrm{x}=0.3[58]$. 


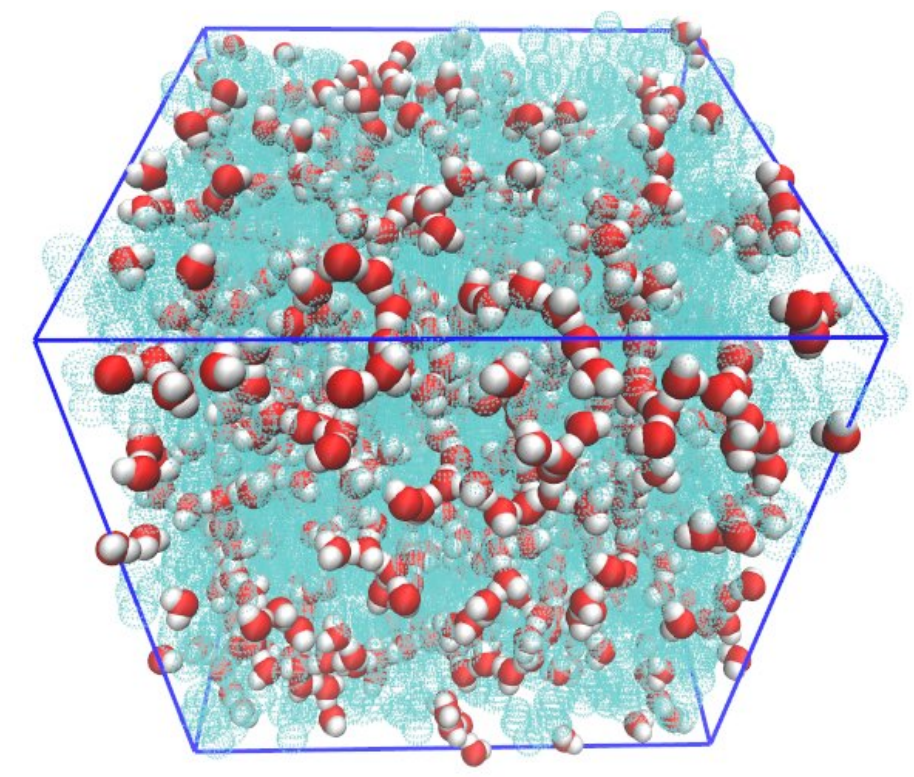

.Fig.2 - Snapshot of the 50\% aqueous DMSO mixture (2048 particles) evidencing linear water clusters. Water molecules are shown as red ball for oxygen atom and white for hydrogen atoms. DMSO is shown in cyan semi-transparent. 

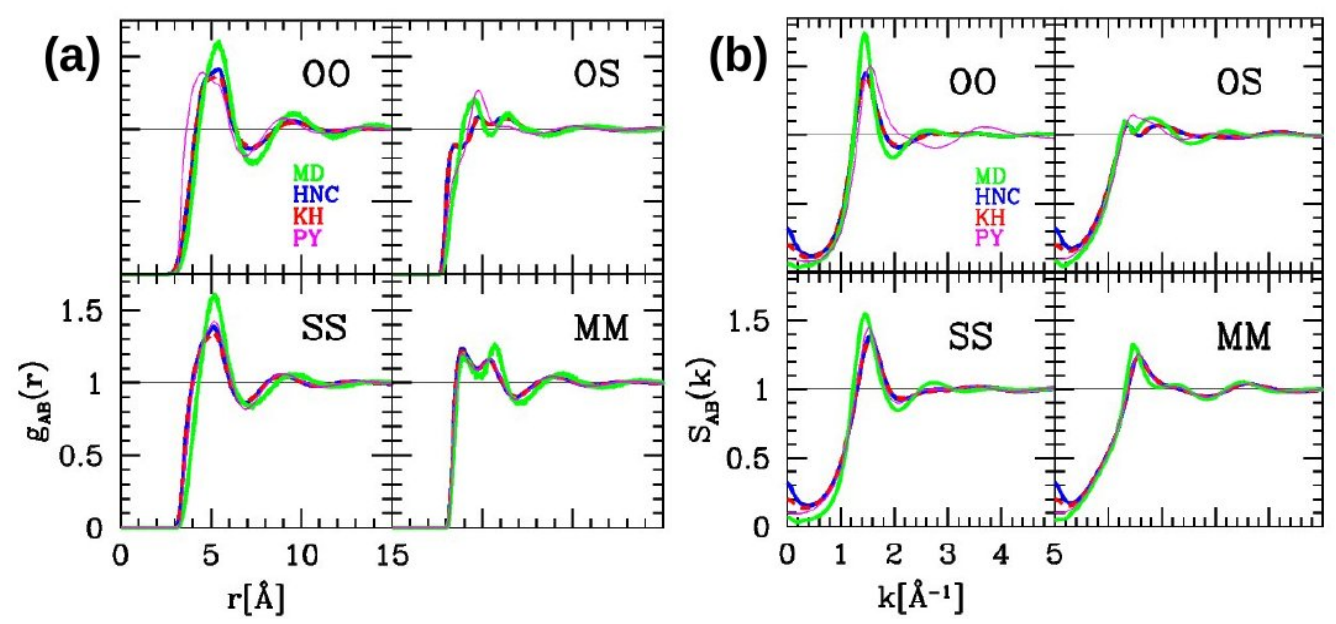

.Fig.3 - Selected atom-atom correlation functions (a) and corresponding structure factors (b) for the neat DMSO mixture, comparing simulation data (green) to HNC(blue), KH(dashed red) and PY(thin magenta) data. The various atom pairs are indicated in the panels. 

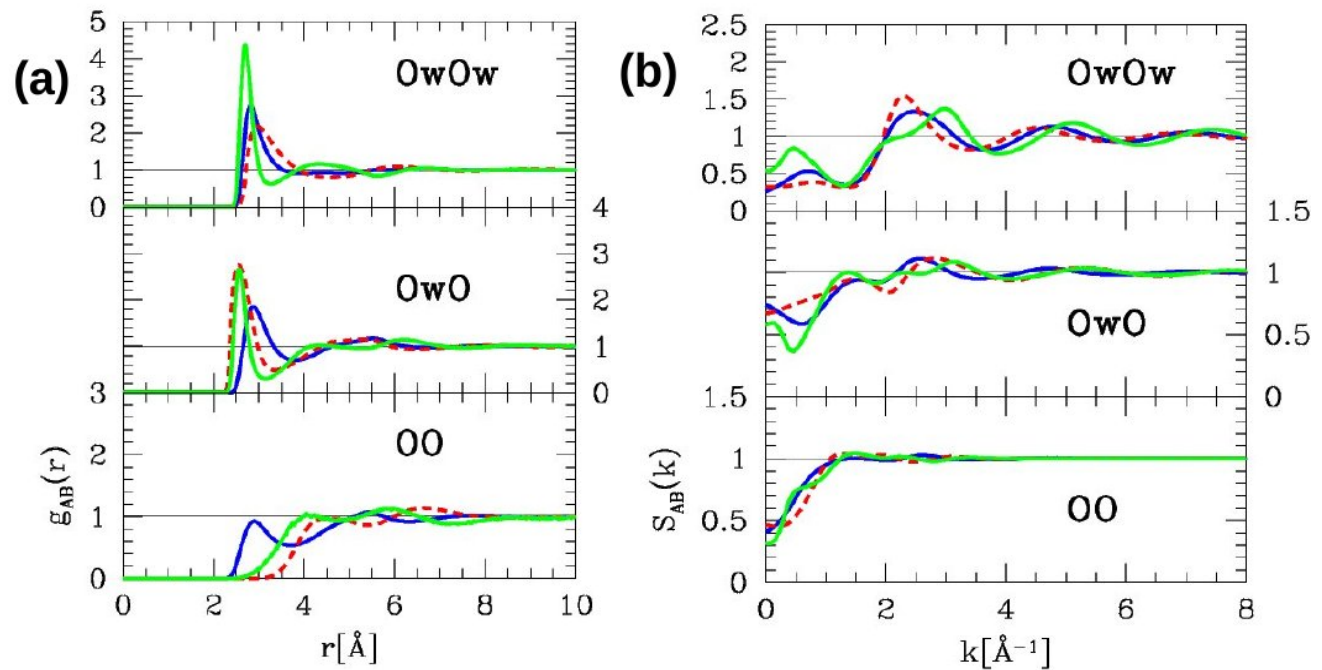

.Fig.4 - Oxygen atom correlation functions (a) and associated structure factors (b) for the $10 \%$ aqueous DMSO mixture. The color conventions are as in Fig.2. 

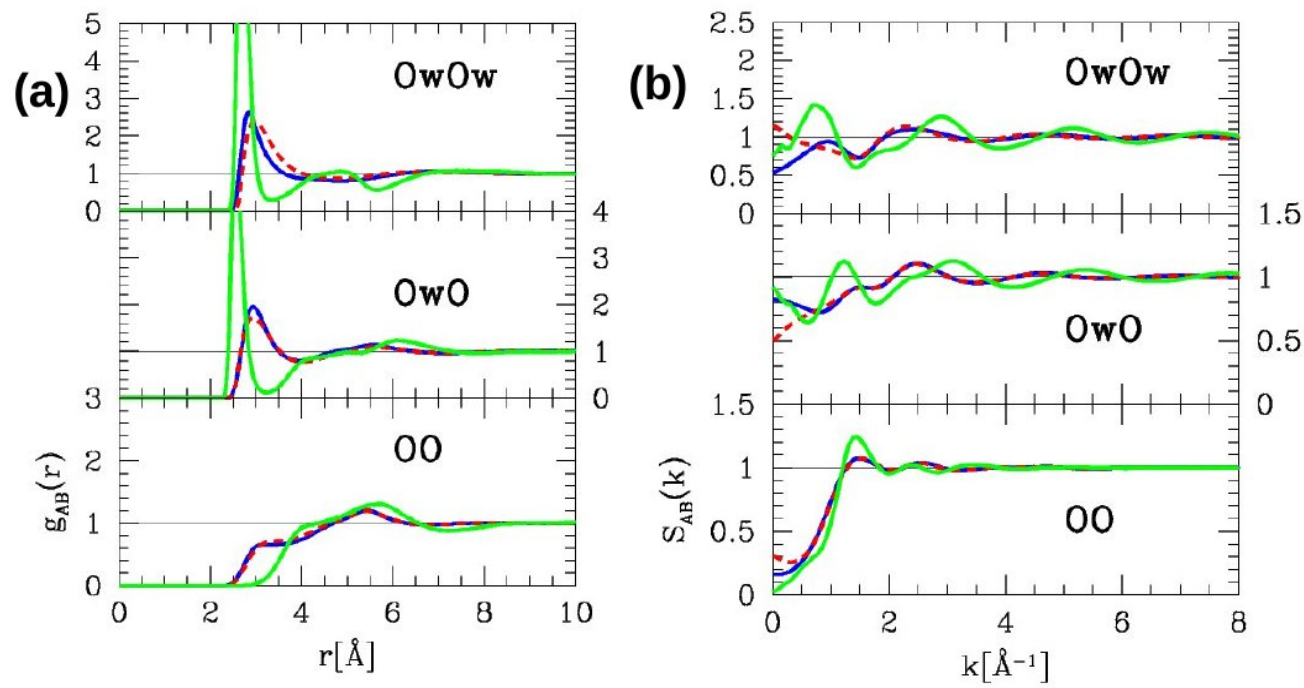

.Fig.5 - Oxygen atom correlation functions (a) and associated structure factors (b) for the 50\% aqueous DMSO mixture. The color conventions are as in Fig.2. 

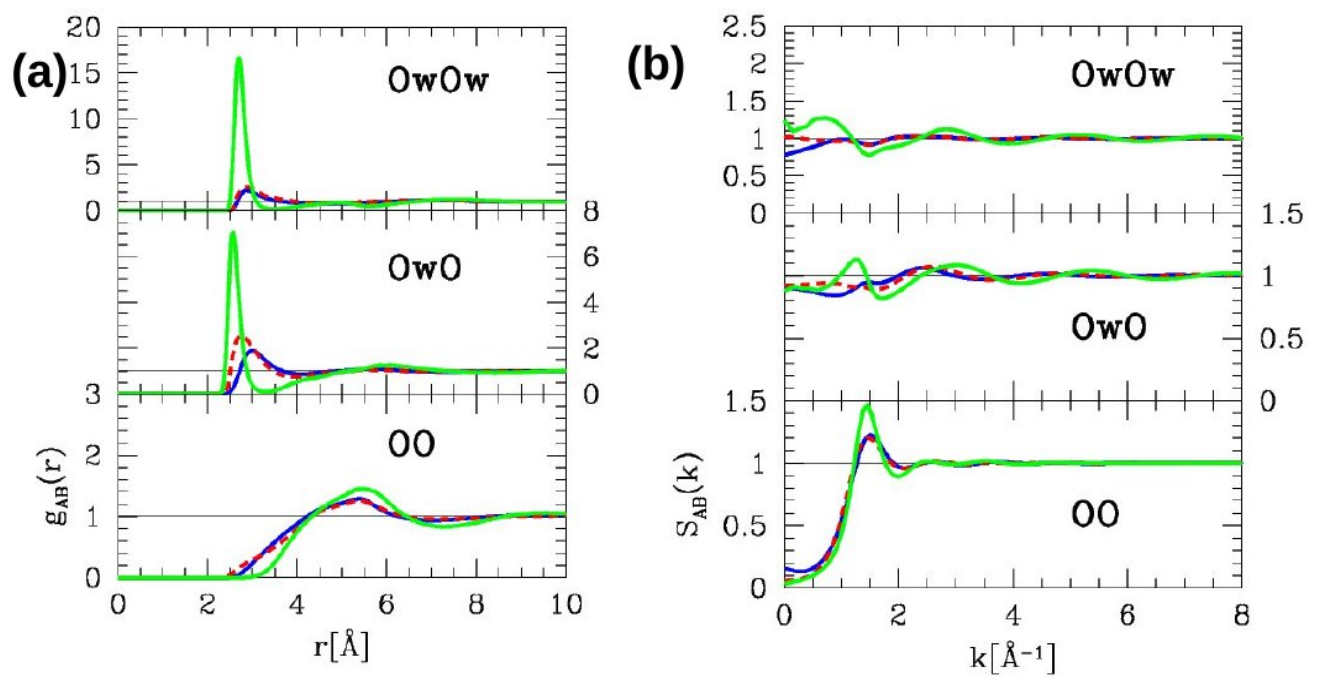

.Fig.6 - Oxygen atom correlation functions (a) and associated structure factors (b) for the $80 \%$ aqueous DMSO mixture. The color conventions are as in Fig.2. 


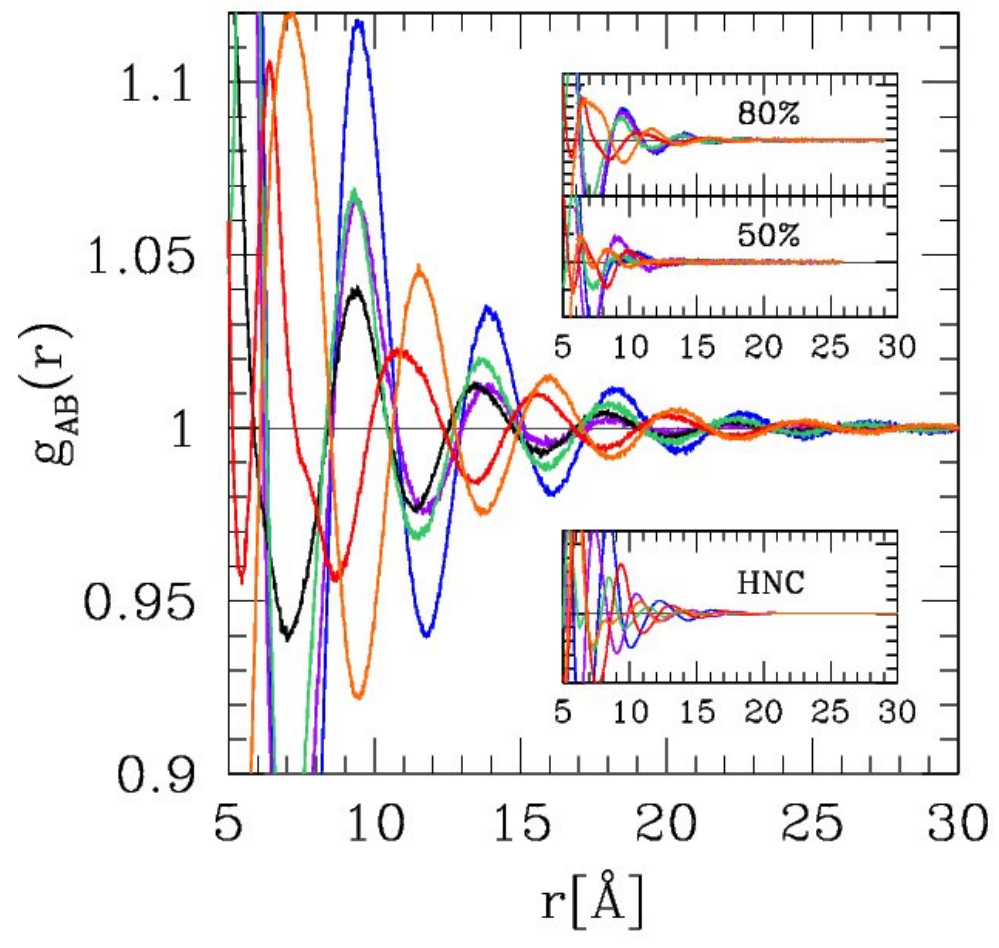

.Fig.7 - DMSO atom-atom correlation functions demonstrating the long range charge order behaviour (see text). $g_{O O}(r)$ in blue, $g_{S S}(r)$ in purple, $g_{S M}(r)$ in black, $g_{M M}(r)$ in green, $g_{O S}(r)$ in red and $g_{O M}(r)$ in orange. The main panel is for neat DMSO, the upper two insets for $80 \%$ and $50 \%$ aqueous DMSO mixtures, and the lower inset for the HNC results. 


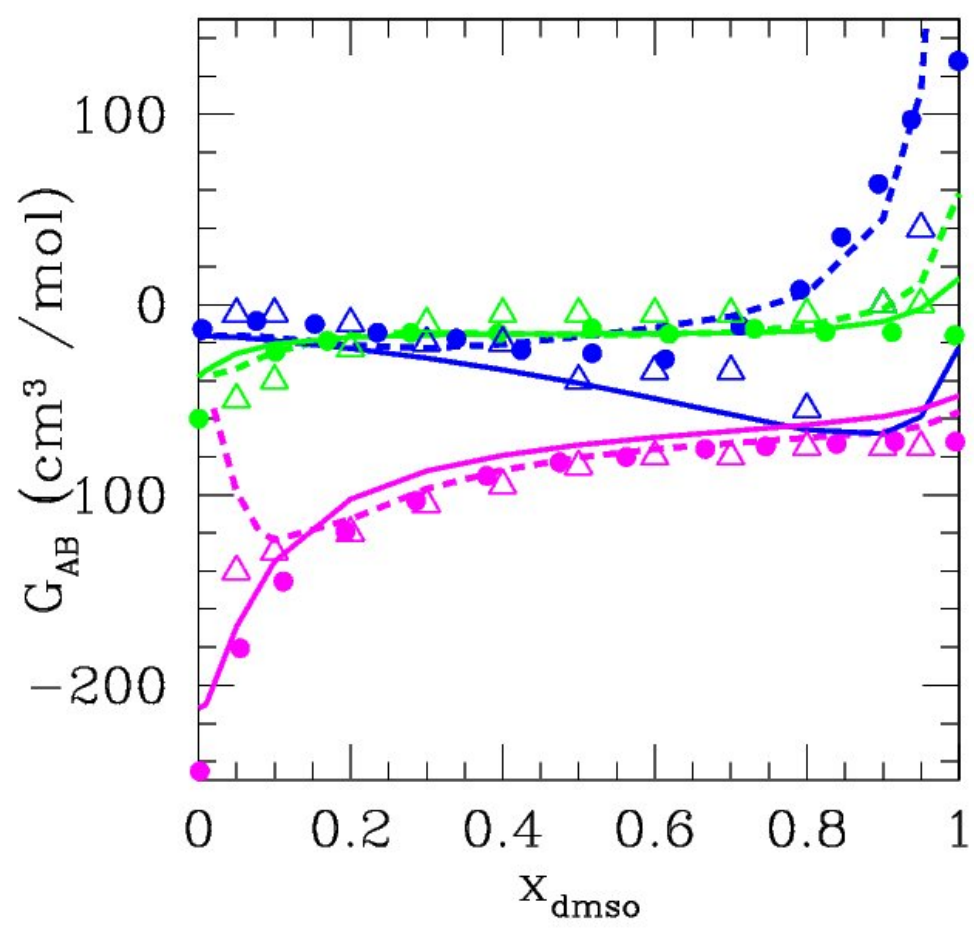

.Fig.8 - Kirkwood-Buff integrals of the aqueous-DMSO mixtures. $G_{W W}$ in blue, $G_{D D}$ in green and $G_{W D}$ in magenta. Filled dots are experimental results from Ref.[28], open triangles simulation results from Ref.[20], blue curve for HNC and dashed curves for $\mathrm{KH}$. 

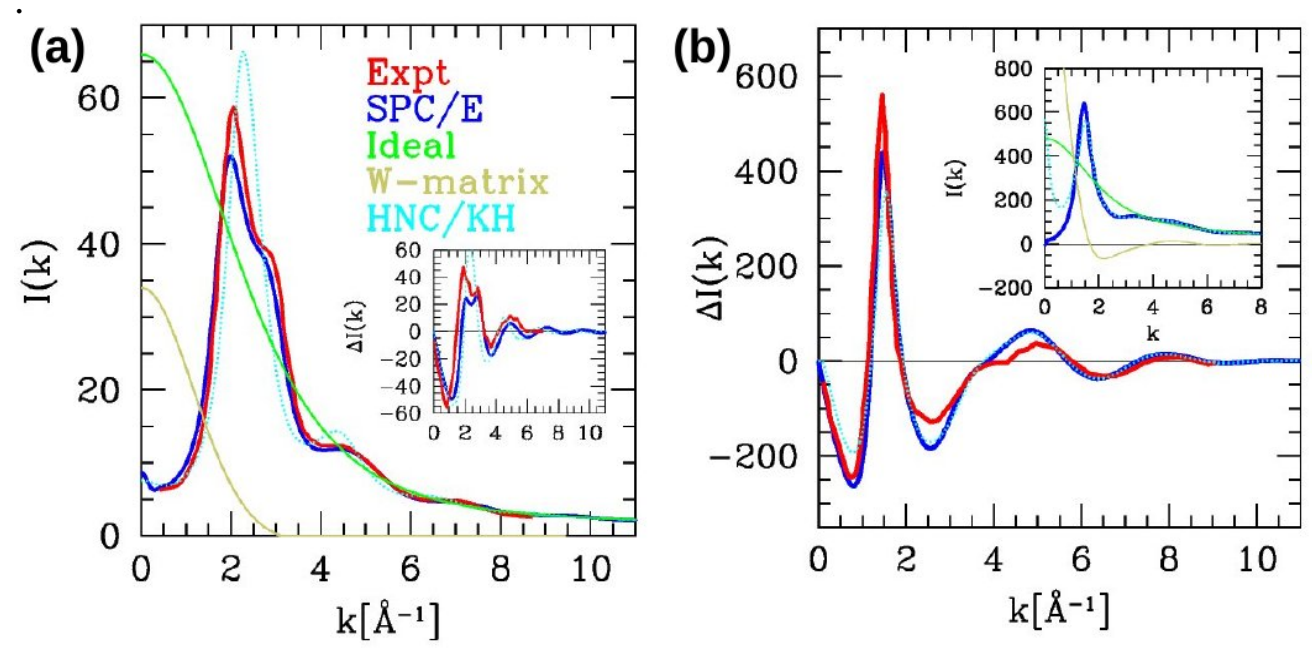

.Fig.9 - Xray scattering intensities $I(k)$ for neat water (a) and neat DMSO (b), together with the $\Delta I(k)$ representations from Eq. 14. Experimental results in red, simulation in blue, IET results in dotted cyan. The ideal contribution from Eq.(11) is in green and the intra-molecular from Eq.(12) contribution in jade. For water, the experimental data for $I(k)$ is from Ref.[57], and $\Delta I(k)$ for both water and DMSO are from from Ref.[22]. 
(a)

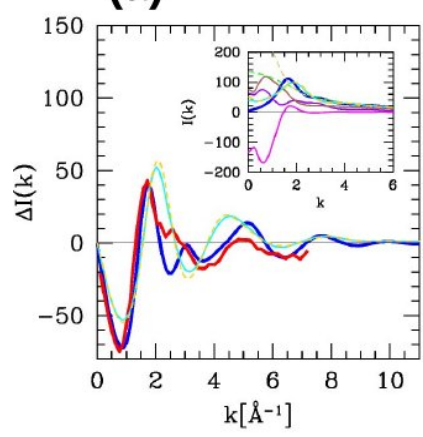

(b)

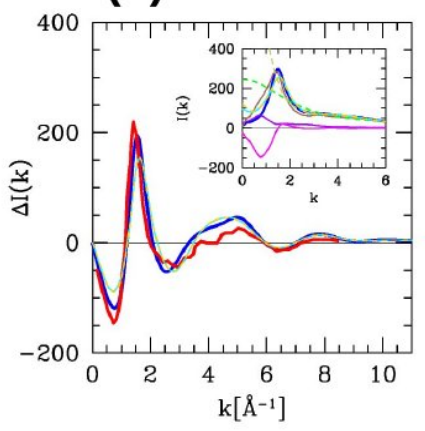

(c)

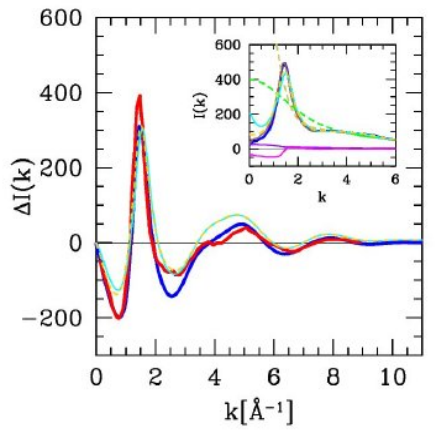

.Fig.10 - Xray scattering intensities $\Delta I(k)$ (main panel) and $I(k)$ (inset) for aqueous mixtures, with $17 \%$ (a), 50\% (b) and 80\% (c) DMSO contents. Experimental results (from Ref.[22]) in red, simulation in blue, HNC results in cyan, and $\mathrm{KH}$ in dashed jade. The ideal and intra-molecular contributions are shown in the insets in dashed green and jade, respectively. The purple curve represents the water-water contribution to $\mathrm{I}(\mathrm{k})$, while the magenta curve is for the cross waterDMSO contribution (see text)) 